\title{
LIST OF LAND SNAILS IN JAVA AND SEVERAL ADJACENT ISLANDS
}

\author{
Ayu Savitri Nurinsiyah \\ Museum Zoologicum Bogoriense. Research Center for Biology, \\ National Research and Innovation Agency (BRIN), \\ Jl. Raya Jakarta-Bogor Km. 46, Cibinong, Bogor 16911, Indonesia \\ e-mail: ayus002@lipi.go.id; ayu_nurinsiyah@yahoo.com
}

Received: 4 December 2021; Accepted: 20 December 2021; Published: 30 December 2021

\begin{abstract}
The malacofauna of Java has been most studied among the Indonesian islands, but the list of land snails in the area remains outdated. This study presents an updated check list of land snails in Java and its adjacent islands. This list is a compilation data from field work in Java conducted in 2013-2016, records from various museums in Europe and Indonesia, collections from private collectors, data from citizen sciences, and literatures. In total, 263 land snail species were recorded in Java and its adjacent islands. The number comprises of 36 families i.e. Subclass Neritimorpha (2 families), Caenogastropoda (6 families), and Heterobranchia (28 families). About $40 \%$ are species endemic to Java and among them have restricted distribution to certain areas. In addition, 5\% or 13 introduced species were recorded in Java.
\end{abstract}

Key words: biodiversity, Gastropoda, Indonesia, Mollusca, terrestrial

\section{INTRODUCTION}

Java is among the main islands in Indonesia which covers $129 \mathrm{~km}^{2}$ or $6.7 \%$ of the whole of archipelago (BPS, 2017). Administratively, Java is divided into six provinces: Banten, DKI Jakarta, West Java, Central Java, DI Yogyakarta, and East Java. It is located between $6^{\circ}$ $37^{\prime} 18^{\prime \prime} \mathrm{S}$ (Panaitan Island, Banten) to $7^{\circ} 11^{\prime} 18^{\prime \prime} \mathrm{S}$ (Kangean Islands, East Java) and $112^{\circ}$ 38'47” E (Bawean Island, East Java) to 114³1'34” E (Alas Purwo, East Java). The island is covered by volcanic features, alluvial sediments, and areas of uplifted coral limestone. During the Quaternary period, the size of Java's land coverage changed due to the sea-level fluctuations. Nevertheless, the lowland and montane forest in the area were larger in the Last Glacial Maximum (LGM) compared to the present condition (Cannon et al., 2009). The forest present today in Sumatra, Borneo, Peninsular Malaysia, and Java are believed to be refugia for the region's biodiversity.

Java is the most populous island in the world and, accordingly, most of its area has been strongly modified by man. The island has suffered massive land use change throughout time. The area was divided into several land use types where only $5 \%$ of the island is covered by natural forest (Whitten et al., 1997; Purba et al., 2014). Forest in Java covers a total of $3,086,200$ ha, consisting of 74,100 ha of primary forest, 788,200 ha of secondary forest and 2,240,000 ha of plantations (KLHK, 2016). The greatest threat to forest areas in Java today comes from anthropogenic factors, i.e. land conversions (forest destruction). In addition to 
habitat loss, the existence of forest inhabitants is also endangered by hunting/trade, invasive alien species, and climate change (Hughes, 2017).

There are 27,474 described land snails in the world (Molluscabase.org, 2021). Yet, the scientific knowledge of the species diversity is scarce. Land snail species tend to have very small ranges, sensitive to changes associated with human disturbance, and are, thus, especially prone to extinction by habitat destruction (Schilthuizen et al., 2005; Douglas et al., 2013).

The work of land snails in Java is the most complete compared to other islands in the Indonesian archipelago. The earliest recorded discovery of land snail in Java was conducted by Johan Coenraad van Hasselt (JC van Hasselt) in 1821-1823 and recorded 40 land snail species (Martens, 1867). The most comprehensive systematic work on land snails in Java was by Möllendorf (1897) and van Benthem Jutting (1941, 1948, 1950, 1952) who described 71 species and 171 species respectively. In addition, Vermeulen (1996) discovered four new species and added the list.

The number of land snail research in Java are also growing in the $21^{\text {st }}$ century (Dharma, 2005; Heryanto et al., 2003; Heryanto, 2008, 2011, 2012, 2017; Marwoto, 2011; Nurinsiyah, 2015; Nurinsiyah et al., 2016; Nurinsiyah \& Hausdorf, 2019; Mujiono \& Priawandiputra, 2020; Nurinsiyah \& Hausdorf, 2020; Nurhayati et al., 2021). In addition, discoveries of new land snail species in Java are rising (Dharma, 2007, 2014; Nurinsiyah \& Hausdorf, 2017a, 2017b; Greķe, 2019; Nurinsiyah et al., 2019). In this paper, I aim to provide an updated checklist of land snails in Java and its adjacent islands, which administratively belong to the provinces in Java.

\section{MATERIALS AND METHODS}

The study is based on the material collected from field survey in Java and surrounding islands including Sempu and Madura Islands in 2013-2016. The list was also based on the examination of land snail collections from the Museum Zoologicum Bogoriense, Bogor, Indonesia (MZB), the Natural History Museum, London, United Kingdom (NHM), the Naturalis Biodiversity Center, formerly Rijksmuseum van Natuurlijke Historie, Leiden, The Netherlands (RMNH), the Senckenberg Museum, Frankfurt, Germany (SMF), the former Zoologisch Museum, Amsterdam, The Netherlands (ZMA; now in the RMNH), the Museum für Naturkunde, Berlin, Germany (ZMB), and the Zoological Museum of the University of Hamburg, Germany (ZMH). Finally, the list was completed with literature study. The checklist covers land snails from the Java mainland as well as its adjacent islands for instance the Panaitan, Peucang, and Dua islands (Banten), thousand islands in Jakarta Bay, Nusa Kambangan (Central Java), Sempu, Nusa Barung, Madura, Bawean and Kangean islands 
(East Java). I excluded the list of land snails from Krakatau Islands because administratively they belong to Lampung Province (Sumatra).

Identification and validation processes referred to van Benthem Jutting (1948, 1950, 1952), Loosjes (1953), Butot (1955), Winter (1983), Dharma (1992, 2007, 2014), Winter \& Vermeulen (1998), Vermeulen \& Whitten (1998), Gomes \& Thomé (2004), Heryanto (2011), and Páll-Gergely et al. (2020). The systematic arrangement and taxonomy status of the determined gastropods follows the classification of Bouchet et al. (2017) and Molluscabase.org.

\section{RESULTS}

A total of 263 land snail species of 36 families, i.e. Subclass Neritimorpha (2 families), Caenogastropoda (6 families), and Heterobranchia (28 families) were recorded in Java and adjacent islands (Table 1). Among them, 246 species were recorded in the main island of Java. Seventeen species were recorded only in the adjacent islands and not on the main island of Java. About 40\% (104 species) of the 263 land snail species recorded were endemic to Java and its adjacent islands.

Table 1. Land snail of Java (*endemic species to Java and its adjacent islands; **introduced species to Java) The presence is indicated with $(+)$

\begin{tabular}{|c|c|c|c|c|c|}
\hline \multirow[b]{2}{*}{ No } & \multirow[b]{2}{*}{ Subclass } & \multirow[b]{2}{*}{ Family } & \multirow[b]{2}{*}{ Species } & \multicolumn{2}{|c|}{ Distribution } \\
\hline & & & & Java & $\begin{array}{c}\text { Adjacent } \\
\text { Islands }\end{array}$ \\
\hline 1 & Neritimorpha & Helicinidae & $\begin{array}{l}\text { Geophorus oxytropis } \\
\text { (Gray, 1839) }\end{array}$ & + & \\
\hline 2 & Neritimorpha & Helicinidae & $\begin{array}{l}\text { Geophorus rollei } \\
\text { (Sykes, 1901)* }\end{array}$ & & + \\
\hline 3 & Neritimorpha & Helicinidae & $\begin{array}{l}\text { Sulfurina biconical } \\
\text { (Martens, 1867) }\end{array}$ & + & \\
\hline 4 & Neritimorpha & Hydrocenidae & $\begin{array}{l}\text { Georissa javana } \\
\text { Möllendorff, } 1897\end{array}$ & + & + \\
\hline 5 & Neritimorpha & Hydrocenidae & $\begin{array}{l}\text { Georissa laeviuscula } \\
\text { Möllendorff, } 1897\end{array}$ & + & \\
\hline 6 & Caenogastropoda & Alycaeidae & $\begin{array}{l}\text { Chamalycaeus fruhstorferi } \\
\text { (Möllendorff, 1897)* }\end{array}$ & + & + \\
\hline 7 & Caenogastropoda & Alycaeidae & $\begin{array}{l}\text { Chamalycaeus reticulatus } \\
\text { (Möllendorff, 1897)* }\end{array}$ & + & \\
\hline 8 & Caenogastropoda & Alycaeidae & $\begin{array}{l}\text { Dicharax candrakirana } \\
\text { Nurinsiyah \& Hausdorf, 2017* }\end{array}$ & & + \\
\hline 9 & Caenogastropoda & Alycaeidae & $\begin{array}{l}\text { Dicharax longituba } \\
\text { (Martens, 1864) }\end{array}$ & + & \\
\hline 10 & Caenogastropoda & Alycaeidae & $\begin{array}{l}\text { Pincerna crenilabris } \\
\text { (Möllendorff, 1897) }\end{array}$ & + & \\
\hline 11 & Caenogastropoda & Alycaeidae & $\begin{array}{l}\text { Stomacosmethis jagori } \\
\text { (Martens, 1860) }\end{array}$ & + & + \\
\hline 12 & Caenogastropoda & Cyclophoridae & $\begin{array}{l}\text { Cyclophorus kibleri } \\
\text { Fulton, } 1907^{*}\end{array}$ & + & \\
\hline 13 & Caenogastropoda & Cyclophoridae & $\begin{array}{l}\text { Cyclophorus perdix } \\
\text { (Broderip \& Sowerby, 1830) }\end{array}$ & + & + \\
\hline 14 & Caenogastropoda & Cyclophoridae & $\begin{array}{l}\text { Cyclophorus rafflesi } \\
\text { (Broderip \& Sowerby, 1830) }\end{array}$ & + & + \\
\hline
\end{tabular}


Table 1. (continued)

\begin{tabular}{|c|c|c|c|c|c|}
\hline \multirow{2}{*}{ No } & \multirow{2}{*}{ Subclass } & \multirow{2}{*}{ Family } & \multirow{2}{*}{ Species } & \multicolumn{2}{|c|}{ Distribution } \\
\hline & & & & Java & $\begin{array}{l}\text { Adjacent } \\
\text { Islands }\end{array}$ \\
\hline 15 & Caenogastropoda & Cyclophoridae & $\begin{array}{l}\text { Cyclotus discoideus } \\
\text { Sowerby, } 1843\end{array}$ & + & \\
\hline 16 & Caenogastropoda & Cyclophoridae & $\begin{array}{l}\text { Cyclotus kangeanus } \\
\text { Schepman, 1909* }\end{array}$ & & + \\
\hline 17 & Caenogastropoda & Cyclophoridae & $\begin{array}{l}\text { Ditropopsis fruhstorferi } \\
\text { (Möllendorff, 1897)* }\end{array}$ & + & \\
\hline 18 & Caenogastropoda & Cyclophoridae & $\begin{array}{l}\text { Japonia ciliocincta } \\
\text { (Martens, 1865) }\end{array}$ & + & \\
\hline 19 & Caenogastropoda & Cyclophoridae & $\begin{array}{l}\text { Japonia trochulus } \\
\text { (Martens, 1867) }\end{array}$ & & + \\
\hline 20 & Caenogastropoda & Cyclophoridae & $\begin{array}{l}\text { Lagocheilus ciliferus } \\
\text { (Mousson, 1849) }\end{array}$ & + & + \\
\hline 21 & Caenogastropoda & Cyclophoridae & $\begin{array}{l}\text { Lagocheilus convexus } \\
\text { Möllendorff, 1897* }\end{array}$ & + & \\
\hline 22 & Caenogastropoda & Cyclophoridae & $\begin{array}{l}\text { Lagocheilus grandipilus } \\
\text { Böttger, } 1891\end{array}$ & + & \\
\hline 23 & Caenogastropoda & Cyclophoridae & $\begin{array}{l}\text { Lagocheilus humilis } \\
\text { Möllendorff, } 1897^{*}\end{array}$ & + & \\
\hline 24 & Caenogastropoda & Cyclophoridae & $\begin{array}{l}\text { Lagocheilus macromphalus } \\
\text { Möllendorff, 1897* }\end{array}$ & + & \\
\hline 25 & Caenogastropoda & Cyclophoridae & $\begin{array}{l}\text { Lagocheilus obliquistriatus } \\
\text { Bullen, } 1904\end{array}$ & + & + \\
\hline 26 & Caenogastropoda & Cyclophoridae & $\begin{array}{l}\text { Leptopoma altum } \\
\text { Möllendorff, } 1897\end{array}$ & + & \\
\hline 27 & Caenogastropoda & Cyclophoridae & $\begin{array}{l}\text { Leptopoma perlucidum } \\
\text { (Grateloup, 1840) }\end{array}$ & + & + \\
\hline 28 & Caenogastropoda & Cyclophoridae & $\begin{array}{l}\text { Opisthoporus corniculus } \\
\text { (Mousson, 1849)* }\end{array}$ & + & + \\
\hline 29 & Caenogastropoda & Cyclophoridae & $\begin{array}{l}\text { Pterocyclos sluiteri } \\
\text { O. Böttger, } 1890^{*}\end{array}$ & + & \\
\hline 30 & Caenogastropoda & Diplommatinidae & $\begin{array}{l}\text { Arinia yanseni } \\
\text { Nurinsiyah \& Hausdorf, } 2017\end{array}$ & + & \\
\hline 31 & Caenogastropoda & Diplommatinidae & $\begin{array}{l}\text { Diplommatina auriculata } \\
\text { Möllendorff, } 1897\end{array}$ & + & + \\
\hline 32 & Caenogastropoda & Diplommatinidae & $\begin{array}{l}\text { Diplommatina baliana } \\
\text { Fulton, } 1899\end{array}$ & + & + \\
\hline 33 & Caenogastropoda & Diplommatinidae & $\begin{array}{l}\text { Diplommatina calcarata } \\
\text { Möllendorff, } 1897^{*}\end{array}$ & + & \\
\hline 34 & Caenogastropoda & Diplommatinidae & $\begin{array}{l}\text { Diplommatina canaliculata } \\
\text { Möllendorff, } 1887\end{array}$ & + & \\
\hline 35 & Caenogastropoda & Diplommatinidae & $\begin{array}{l}\text { Diplommatina cyclostoma } \\
\text { Möllendorff, } 1897 *\end{array}$ & + & \\
\hline 36 & Caenogastropoda & Diplommatinidae & $\begin{array}{l}\text { Diplommatina diplostoma } \\
\text { Rensch, } 1931\end{array}$ & + & \\
\hline 37 & Caenogastropoda & Diplommatinidae & $\begin{array}{l}\text { Diplommatina halimunensis } \\
\text { Nurinsiyah \& Hausdorf, 2017* }\end{array}$ & + & \\
\hline 38 & Caenogastropoda & Diplommatinidae & $\begin{array}{l}\text { Diplommatina heryantoi } \\
\text { Nurinsiyah \& Hausdorf, 2017* }\end{array}$ & + & \\
\hline
\end{tabular}


Table 1. (continued)

\begin{tabular}{|c|c|c|c|c|c|}
\hline \multirow{2}{*}{ No } & \multirow{2}{*}{ Subclass } & \multirow{2}{*}{ Family } & \multirow{2}{*}{ Species } & \multicolumn{2}{|c|}{ Distribution } \\
\hline & & & & Java & $\begin{array}{l}\text { Adjacent } \\
\text { Islands }\end{array}$ \\
\hline 39 & Caenogastropoda & Diplommatinidae & $\begin{array}{l}\text { Diplommatina heteroglypha } \\
\text { van Benthem Jutting, 1948* }\end{array}$ & + & \\
\hline 40 & Caenogastropoda & Diplommatinidae & $\begin{array}{l}\text { Diplommatina javana } \\
\text { Möllendorff, } 1897\end{array}$ & + & \\
\hline 41 & Caenogastropoda & Diplommatinidae & $\begin{array}{l}\text { Diplommatina kakenca } \\
\text { Nurinsiyah \& Hausdorf, 2017* }\end{array}$ & + & \\
\hline 42 & Caenogastropoda & Diplommatinidae & $\begin{array}{l}\text { Diplommatina majapahit } \\
\text { Grekse, 2019* }\end{array}$ & + & \\
\hline 43 & Caenogastropoda & Diplommatinidae & $\begin{array}{l}\text { Diplommatina nevilli } \\
\text { (Crosse, 1879) }\end{array}$ & + & + \\
\hline 44 & Caenogastropoda & Diplommatinidae & $\begin{array}{l}\text { Diplommatina perpusilla } \\
\text { Möllendorff, } 1897^{*}\end{array}$ & + & \\
\hline 45 & Caenogastropoda & Diplommatinidae & $\begin{array}{l}\text { Diplommatina planicollis } \\
\text { Möllendorff, } 1897^{*}\end{array}$ & + & \\
\hline 46 & Caenogastropoda & Diplommatinidae & $\begin{array}{l}\text { Diplommatina ristiae } \\
\text { Nurinsiyah \& Hausdorf, 2017* }\end{array}$ & + & \\
\hline 47 & Caenogastropoda & Diplommatinidae & $\begin{array}{l}\text { Diplommatina sulcicollis } \\
\text { Möllendorff, } 1897^{*}\end{array}$ & + & \\
\hline 48 & Caenogastropoda & Diplommatinidae & $\begin{array}{l}\text { Diplommatina tetragonostoma } \\
\text { Möllendorff, } 1897^{*}\end{array}$ & + & \\
\hline 49 & Caenogastropoda & Diplommatinidae & $\begin{array}{l}\text { Opisthostoma javanicum } \\
\text { van Benthem Jutting, } 1932\end{array}$ & + & + \\
\hline 50 & Caenogastropoda & Diplommatinidae & $\begin{array}{l}\text { Opisthostoma uranoscopium } \\
\text { van Benthem Jutting, 1932* }\end{array}$ & + & \\
\hline 51 & Caenogastropoda & Diplommatinidae & $\begin{array}{l}\text { Palaina gedeana } \\
\text { Möllendorff, } 1897\end{array}$ & + & \\
\hline 52 & Caenogastropoda & Diplommatinidae & $\begin{array}{l}\text { Palaina nubigena } \\
\text { Möllendorff, } 1897 *\end{array}$ & + & \\
\hline 53 & Caenogastropoda & Pupinidae & $\begin{array}{l}\text { Pupina bipalatalis } \\
\text { Böttger, } 1890^{*}\end{array}$ & + & \\
\hline 54 & Caenogastropoda & Pupinidae & $\begin{array}{l}\text { Pupina compacta } \\
\text { Möllendorff, } 1897^{*}\end{array}$ & + & \\
\hline 55 & Caenogastropoda & Pupinidae & $\begin{array}{l}\text { Tylotoechus junghuhni } \\
\text { Martens, } 1867^{*}\end{array}$ & + & \\
\hline 56 & Caenogastropoda & Pupinidae & $\begin{array}{l}\text { Tylotoechus treubi } \\
\text { Böttger, } 1890\end{array}$ & + & \\
\hline 57 & Caenogastropoda & Pupinidae & $\begin{array}{l}\text { Tylotoechus verbeeki } \\
\text { Möllendorff, } 1897^{*}\end{array}$ & + & \\
\hline 58 & Caenogastropoda & Assimineidae & $\begin{array}{l}\text { Anaglyphula tiluana } \\
\text { (Möllendorff, 1897)* }\end{array}$ & + & \\
\hline 59 & Caenogastropoda & Assimineidae & $\begin{array}{l}\text { Omphalotropis columellaris } \\
\text { Quadras \& Möllendorff, } 1893\end{array}$ & + & + \\
\hline 60 & Heterobranchia & Ellobiidae & $\begin{array}{l}\text { Carychium javanum } \\
\text { Möllendorff, } 1897\end{array}$ & + & + \\
\hline 61 & Heterobranchia & Ellobiidae & $\begin{array}{l}\text { Auriculastra semiplicata } \\
\text { (Adams \& Adams, 1854) }\end{array}$ & + & \\
\hline 62 & Heterobranchia & Ellobiidae & $\begin{array}{l}\text { Ellobium aurisjudae } \\
\text { (Linnaeus, 1758) }\end{array}$ & + & + \\
\hline
\end{tabular}


Table 1. (continued)

\begin{tabular}{|c|c|c|c|c|c|}
\hline \multirow{2}{*}{ No } & \multirow{2}{*}{ Subclass } & \multirow{2}{*}{ Family } & \multirow{2}{*}{ Species } & \multicolumn{2}{|c|}{ Distribution } \\
\hline & & & & Java & $\begin{array}{c}\text { Adjacent } \\
\text { Islands }\end{array}$ \\
\hline 63 & Heterobranchia & Ellobiidae & $\begin{array}{l}\text { Ellobium aurismidae } \\
\text { (Linnaeus, 1758) }\end{array}$ & + & + \\
\hline 64 & Heterobranchia & Ellobiidae & $\begin{array}{l}\text { Ellobium tornatelliforme } \\
\text { (Petit de la Saussaye, 1843) }\end{array}$ & + & \\
\hline 65 & Heterobranchia & Ellobiidae & $\begin{array}{l}\text { Ellobium scheepmakeri } \\
\text { (Petit de la Saussaye, 1850) }\end{array}$ & + & + \\
\hline 66 & Heterobranchia & Ellobiidae & $\begin{array}{l}\text { Melampus castaneus } \\
\text { Megerle von Mühlfeld, } 1816\end{array}$ & + & \\
\hline 67 & Heterobranchia & Ellobiidae & $\begin{array}{l}\text { Melampus cumingianus } \\
\text { (Récluz, 1846) }\end{array}$ & + & + \\
\hline 68 & Heterobranchia & Ellobiidae & $\begin{array}{l}\text { Melampus fasciatus } \\
\text { (Deshayes, 1830) }\end{array}$ & + & + \\
\hline 69 & Heterobranchia & Ellobiidae & $\begin{array}{l}\text { Melampus granifer } \\
\text { (Mousson, 1849) }\end{array}$ & + & \\
\hline 70 & Heterobranchia & Ellobiidae & $\begin{array}{l}\text { Melampus luteus } \\
\text { (Quoy \& Gaimard, 1832) }\end{array}$ & + & + \\
\hline 71 & Heterobranchia & Ellobiidae & $\begin{array}{l}\text { Cassidula aurisfelis } \\
\text { (Bruguière, 1789) }\end{array}$ & + & + \\
\hline 72 & Heterobranchia & Ellobiidae & $\begin{array}{l}\text { Cassidula faba } \\
\text { (Pfeiffer, 1853) }\end{array}$ & + & \\
\hline 73 & Heterobranchia & Ellobiidae & $\begin{array}{l}\text { Cassidula nucleus } \\
\text { (Gmelin, 1791) }\end{array}$ & + & + \\
\hline 74 & Heterobranchia & Ellobiidae & $\begin{array}{l}\text { Cassidula sowerbyana } \\
\text { (Pfeiffer, 1853) }\end{array}$ & + & \\
\hline 75 & Heterobranchia & Ellobiidae & $\begin{array}{l}\text { Cassidula sulculosa } \\
\text { (Mousson, 1849) }\end{array}$ & & + \\
\hline 76 & Heterobranchia & Ellobiidae & $\begin{array}{l}\text { Laemodonta monilifera } \\
\text { (Adams \& Adams, 1854) }\end{array}$ & & + \\
\hline 77 & Heterobranchia & Ellobiidae & $\begin{array}{l}\text { Laemodonta siamensis } \\
\text { (Morelet, 1875) }\end{array}$ & + & \\
\hline 78 & Heterobranchia & Ellobiidae & $\begin{array}{l}\text { Laemodonta typica } \\
\text { (Adams \& Adams, 1854) }\end{array}$ & + & \\
\hline 79 & Heterobranchia & Ellobiidae & $\begin{array}{l}\text { Pythia castanea } \\
\text { (Lesson, 1831) }\end{array}$ & & + \\
\hline 80 & Heterobranchia & Ellobiidae & $\begin{array}{l}\text { Pythia imperforata } \\
\text { (Adams, 1850) }\end{array}$ & + & + \\
\hline 81 & Heterobranchia & Ellobiidae & $\begin{array}{l}\text { Pythia pantherina } \\
\text { (Adams, 1850) }\end{array}$ & + & + \\
\hline 82 & Heterobranchia & Ellobiidae & $\begin{array}{l}\text { Pythia plicata } \\
\text { (Gray, 1825) }\end{array}$ & + & + \\
\hline 83 & Heterobranchia & Ellobiidae & $\begin{array}{l}\text { Pythia scarabaeus } \\
\text { (Linnaeus, 1758) }\end{array}$ & + & + \\
\hline 84 & Heterobranchia & Ellobiidae & $\begin{array}{l}\text { Pythia trigona } \\
\text { (Troschel, 1838) }\end{array}$ & + & + \\
\hline 85 & Heterobranchia & Ellobiidae & $\begin{array}{l}\text { Pythia undata } \\
\text { (Lesson, 1831) }\end{array}$ & + & + \\
\hline 86 & Heterobranchia & Veronicellidae & $\begin{array}{l}\text { Filicaulis bleekeri } \\
\text { (Keferstein, 1865) }\end{array}$ & + & \\
\hline
\end{tabular}


Table 1. (continued)

\begin{tabular}{|c|c|c|c|c|c|}
\hline \multirow{2}{*}{ No } & \multirow{2}{*}{ Subclass } & \multirow{2}{*}{ Family } & \multirow{2}{*}{ Species } & \multicolumn{2}{|c|}{ Distribution } \\
\hline & & & & Java & $\begin{array}{l}\text { Adjacent } \\
\text { Islands }\end{array}$ \\
\hline 87 & Heterobranchia & Veronicellidae & $\begin{array}{l}\text { Laevicaulis alte } \\
\text { (Férussac, 1822)** }\end{array}$ & + & \\
\hline 88 & Heterobranchia & Veronicellidae & $\begin{array}{l}\text { Semperula maculata } \\
\text { (Templeton, 1858) }\end{array}$ & + & + \\
\hline 89 & Heterobranchia & Veronicellidae & $\begin{array}{l}\text { Semperula wallacei } \\
\text { (Issel, 1874) }\end{array}$ & + & \\
\hline 90 & Heterobranchia & Veronicellidae & $\begin{array}{l}\text { Valiguna siamensis } \\
\text { (Martens, 1867)* }\end{array}$ & + & + \\
\hline 91 & Heterobranchia & Rathouisiidae & $\begin{array}{l}\text { Atopos ouwensi } \\
\text { Collinge, } 1908^{*}\end{array}$ & + & \\
\hline 92 & Heterobranchia & Achatinidae & $\begin{array}{l}\text { Allopeas clavulinum } \\
\text { (Potiez \& Michaud, 1838)** }\end{array}$ & + & + \\
\hline 93 & Heterobranchia & Achatinidae & $\begin{array}{l}\text { Allopeas gracile } \\
\text { (Hutton, 1834)** }\end{array}$ & + & + \\
\hline 94 & Heterobranchia & Achatinidae & $\begin{array}{l}\text { Geostilbia aperta } \\
\text { (Swainson, 1840)** }\end{array}$ & + & + \\
\hline 95 & Heterobranchia & Achatinidae & $\begin{array}{l}\text { Glessula sumatrana } \\
\text { (Martens, 1864) }\end{array}$ & + & \\
\hline 96 & Heterobranchia & Achatinidae & $\begin{array}{l}\text { Lissachatina fulica } \\
\text { (Bowdich, 1822)** }\end{array}$ & + & + \\
\hline 97 & Heterobranchia & Achatinidae & $\begin{array}{l}\text { Paropeas achatinaceum } \\
\text { (Pfeiffer, 1846) }\end{array}$ & + & + \\
\hline 98 & Heterobranchia & Achatinidae & $\begin{array}{l}\text { Paropeas acutissimum } \\
\text { (Mousson, 1857) }\end{array}$ & + & \\
\hline 99 & Heterobranchia & Achatinidae & $\begin{array}{l}\text { Subulina octona } \\
\text { (Bruguière, 1789)** }\end{array}$ & + & + \\
\hline 100 & Heterobranchia & Streptaxidae & $\begin{array}{l}\text { Gulella bicolor } \\
\text { (Hutton, 1834)** }\end{array}$ & + & + \\
\hline 101 & Heterobranchia & Charopidae & $\begin{array}{l}\text { Corinomala baliana } \\
\text { (Rensch, 1930) }\end{array}$ & + & \\
\hline 102 & Heterobranchia & Charopidae & $\begin{array}{l}\text { Discocharopa aperta } \\
\text { (Möllendorff, 1888) }\end{array}$ & + & + \\
\hline 103 & Heterobranchia & Charopidae & $\begin{array}{l}\text { Philalanka micromphala } \\
\text { van Benthem Jutting, } 1952\end{array}$ & + & \\
\hline 104 & Heterobranchia & Charopidae & $\begin{array}{l}\text { Philalanka nannophya } \\
\text { Rensch, } 1932\end{array}$ & + & + \\
\hline 105 & Heterobranchia & Charopidae & $\begin{array}{l}\text { Philalanka setifera } \\
\text { Vermeulen, } 1996\end{array}$ & + & \\
\hline 106 & Heterobranchia & Charopidae & $\begin{array}{l}\text { Philalanka thienemanni } \\
\text { Rensch, } 1932\end{array}$ & + & + \\
\hline 107 & Heterobranchia & Charopidae & $\begin{array}{l}\text { Philalanka tjibodasensis } \\
\text { (Leschke, 1914) }\end{array}$ & + & \\
\hline 108 & Heterobranchia & Charopidae & $\begin{array}{l}\text { Thysanota conula } \\
\text { (Blanford, 1865) }\end{array}$ & + & \\
\hline 109 & Heterobranchia & Endodontidae & $\begin{array}{l}\text { Beilania philippinensis } \\
\text { (Semper, 1874) }\end{array}$ & + & \\
\hline 110 & Heterobranchia & Helicodiscidae & $\begin{array}{l}\text { Stenopylis coarctata } \\
\text { (Möllendorff, 1894) }\end{array}$ & + & + \\
\hline
\end{tabular}


Table 1. (continued)

\begin{tabular}{|c|c|c|c|c|c|}
\hline \multirow{2}{*}{ No } & \multirow{2}{*}{ Subclass } & \multirow{2}{*}{ Family } & \multirow{2}{*}{ Species } & \multicolumn{2}{|c|}{ Distribution } \\
\hline & & & & Java & $\begin{array}{l}\text { Adjacent } \\
\text { Islands }\end{array}$ \\
\hline 111 & Heterobranchia & Succineidae & $\begin{array}{l}\text { Succinea gracilis } \\
(\text { Lea, } 1841)^{*}\end{array}$ & & + \\
\hline 112 & Heterobranchia & Succineidae & $\begin{array}{l}\text { Succinea listeri } \\
(\text { Smith, 1889)* }\end{array}$ & + & + \\
\hline 113 & Heterobranchia & Succineidae & $\begin{array}{l}\text { Succinea minuta } \\
\text { (Martens, 1867) }\end{array}$ & + & \\
\hline 114 & Heterobranchia & Succineidae & $\begin{array}{l}\text { Succinea obesa } \\
\text { (Martens, 1867) }\end{array}$ & + & + \\
\hline 115 & Heterobranchia & Achatinellidae & $\begin{array}{l}\text { Elasmias manilense } \\
\text { (Dohrn, 1863) }\end{array}$ & + & + \\
\hline 116 & Heterobranchia & Achatinellidae & $\begin{array}{l}\text { Elasmias sundanum } \\
\text { (Möllendorff, 1897) }\end{array}$ & + & \\
\hline 117 & Heterobranchia & Achatinellidae & $\begin{array}{l}\text { Lamellidea cylindrica } \\
\text { (Sykes, 1900) }\end{array}$ & & + \\
\hline 118 & Heterobranchia & Achatinellidae & $\begin{array}{l}\text { Lamellidea subcylindrica } \\
\text { (Möllendorff \& Quadras, 1894) }\end{array}$ & + & + \\
\hline 119 & Heterobranchia & Achatinellidae & $\begin{array}{l}\text { Truncatella guerinii } \\
\text { Villa \& Villa, } 1841\end{array}$ & + & + \\
\hline 120 & Heterobranchia & Pupillidae & $\begin{array}{l}\text { Pupoides coenopictus } \\
\text { (Hutton, 1834) }\end{array}$ & & + \\
\hline 121 & Heterobranchia & Cerastidae & $\begin{array}{l}\text { Rhachis zonulata } \\
\text { (Pfeiffer, 1846)** }\end{array}$ & + & + \\
\hline 122 & Heterobranchia & Enidae & $\begin{array}{l}\text { Apoecus alticola } \\
\text { (Dharma, 1996)* }^{*}\end{array}$ & + & \\
\hline 123 & Heterobranchia & Enidae & $\begin{array}{l}\text { Apoecus apertus } \\
\text { (Martens, 1863) }\end{array}$ & & + \\
\hline 124 & Heterobranchia & Enidae & $\begin{array}{l}\text { Apoecus glandula } \\
\text { (Mousson, 1848) }\end{array}$ & + & \\
\hline 125 & Heterobranchia & Enidae & $\begin{array}{l}\text { Apoecus prillwitzi } \\
\text { (Möllendorff, 1897)* }\end{array}$ & + & \\
\hline 126 & Heterobranchia & Enidae & $\begin{array}{l}\text { Apoecus tenggericus } \\
\text { (Möllendorff, 1897) }\end{array}$ & + & \\
\hline 127 & Heterobranchia & Enidae & $\begin{array}{l}\text { Apoecus tenuiliratus } \\
\text { (Möllendorff, 1897)* }\end{array}$ & + & \\
\hline 128 & Heterobranchia & Enidae & $\begin{array}{l}\text { Apoecus thraustus } \\
\text { (Möllendorff, 1897) }\end{array}$ & + & \\
\hline 129 & Heterobranchia & Gastrocoptidae & $\begin{array}{l}\text { Gastrocopta euryomphala } \\
\text { Pilsbry, } 1934\end{array}$ & & + \\
\hline 130 & Heterobranchia & Gastrocoptidae & $\begin{array}{l}\text { Gastrocopta pediculus } \\
\text { (Shuttleworth, 1852) }\end{array}$ & + & + \\
\hline 131 & Heterobranchia & Gastrocoptidae & $\begin{array}{l}\text { Gastrocopta recondita } \\
\text { (Tapparone-Canefri, 1883) }\end{array}$ & + & \\
\hline 132 & Heterobranchia & Gastrocoptidae & $\begin{array}{l}\text { Gastrocopta servilis } \\
\text { (Gould, 1843)** }\end{array}$ & + & + \\
\hline 133 & Heterobranchia & Gastrocoptidae & $\begin{array}{l}\text { Gyliotrachela fruhstorferi } \\
\text { (Möllendorff, 1897)* }\end{array}$ & + & \\
\hline 134 & Heterobranchia & Gastrocoptidae & $\begin{array}{l}\text { Paraboysidia boettgeri } \\
\text { (Möllendorff, 1897) }\end{array}$ & + & \\
\hline
\end{tabular}


Table 1. (continued)

\begin{tabular}{|c|c|c|c|c|c|}
\hline \multirow{2}{*}{ No } & \multirow{2}{*}{ Subclass } & \multirow{2}{*}{ Family } & \multirow{2}{*}{ Species } & \multicolumn{2}{|c|}{ Distribution } \\
\hline & & & & Java & $\begin{array}{l}\text { Adjacent } \\
\text { Islands }\end{array}$ \\
\hline 135 & Heterobranchia & Pyramidulidae & $\begin{array}{l}\text { Pyramidula javana } \\
\text { (Möllendorff, 1897)* }\end{array}$ & + & \\
\hline 136 & Heterobranchia & Truncatellinidae & $\begin{array}{l}\text { Truncatellina insulivaga } \\
\text { (Pilsbry \& Hirase, 1904)* }\end{array}$ & + & \\
\hline 137 & Heterobranchia & Valloniidae & $\begin{array}{l}\text { Pupisoma perpusillum } \\
\text { (Möllendorff, 1897)* }\end{array}$ & + & \\
\hline 138 & Heterobranchia & Valloniidae & $\begin{array}{l}\text { Pupisoma circumlitum } \\
\text { Hedley, } 1897\end{array}$ & + & + \\
\hline 139 & Heterobranchia & Valloniidae & $\begin{array}{l}\text { Pupisoma dioscoricola } \\
\text { (Adams, 1845) }\end{array}$ & + & + \\
\hline 140 & Heterobranchia & Valloniidae & $\begin{array}{l}\text { Pupisoma moleculina } \\
\text { (van Benthem Jutting, 1940) }\end{array}$ & + & \\
\hline 141 & Heterobranchia & Valloniidae & $\begin{array}{l}\text { Pupisoma orcella } \\
\text { (Stoliczka, 1873) }\end{array}$ & + & \\
\hline 142 & Heterobranchia & Vertiginidae & $\begin{array}{l}\text { Insulipupa malayana } \\
\text { (Issel, 1874) }\end{array}$ & + & + \\
\hline 143 & Heterobranchia & Vertiginidae & $\begin{array}{l}\text { Nesopupa nannodes } \\
\text { (Quadras \& Möllendorff, 1898) }\end{array}$ & + & + \\
\hline 144 & Heterobranchia & Vertiginidae & $\begin{array}{l}\text { Nesopupa novopommerana } \\
\text { Rensch, } 1932\end{array}$ & + & \\
\hline 145 & Heterobranchia & Clausiliidae & $\begin{array}{l}\text { Juttingia fucosa } \\
\text { (Loosjes, 1963)* }\end{array}$ & + & \\
\hline 146 & Heterobranchia & Clausiliidae & $\begin{array}{l}\text { Oospira cornea } \\
\text { (Küster, 1844)* }\end{array}$ & + & + \\
\hline 147 & Heterobranchia & Clausiliidae & $\begin{array}{l}\text { Oospira fruhstorferi } \\
\text { (Möllendorff, 1897)* }\end{array}$ & + & \\
\hline 148 & Heterobranchia & Clausiliidae & $\begin{array}{l}\text { Oospira javana } \\
\text { (Pfeiffer, 1841)* }\end{array}$ & + & + \\
\hline 149 & Heterobranchia & Clausiliidae & $\begin{array}{l}\text { Oospira junghuhni } \\
\text { (Küster, 1844) }\end{array}$ & + & \\
\hline 150 & Heterobranchia & Clausiliidae & $\begin{array}{l}\text { Oospira nubigena } \\
\text { (Möllendorff, 1897)* }\end{array}$ & + & \\
\hline 151 & Heterobranchia & Clausiliidae & $\begin{array}{l}\text { Oospira orientalis } \\
\text { (Pfeiffer, 1842)* }\end{array}$ & + & \\
\hline 152 & Heterobranchia & Clausiliidae & $\begin{array}{l}\text { Oospira salacana } \\
\text { (Böttger, 1890)* }\end{array}$ & + & \\
\hline 153 & Heterobranchia & Clausiliidae & $\begin{array}{l}\text { Oospira schepmani } \\
\text { (Möllendorff, 1897)* }\end{array}$ & + & \\
\hline 154 & Heterobranchia & Clausiliidae & $\begin{array}{l}\text { Phaedusa corticina } \\
\text { (Pfeiffer, 1842) }\end{array}$ & + & + \\
\hline 155 & Heterobranchia & Clausiliidae & $\begin{array}{l}\text { Phaedusa moluccensis } \\
\text { (Martens, 1864) }\end{array}$ & + & \\
\hline 156 & Heterobranchia & Phylomycidae & $\begin{array}{l}\text { Meghimatium bilineatum } \\
\text { (Benson, 1842)* }\end{array}$ & + & \\
\hline 157 & Heterobranchia & Phylomycidae & $\begin{array}{l}\text { Meghimatium striatum } \\
\text { van Hasselt, } 1824\end{array}$ & + & \\
\hline 158 & Heterobranchia & Agriolimacidae & $\begin{array}{l}\text { Deroceras laeve } \\
\text { (Müller, 1774)** }\end{array}$ & + & \\
\hline
\end{tabular}


Table 1. (continued)

\begin{tabular}{|c|c|c|c|c|c|}
\hline \multirow{2}{*}{ No } & \multirow{2}{*}{ Subclass } & \multirow{2}{*}{ Family } & \multirow{2}{*}{ Species } & \multicolumn{2}{|c|}{ Distribution } \\
\hline & & & & Java & $\begin{array}{l}\text { Adjacent } \\
\text { Islands }\end{array}$ \\
\hline 159 & Heterobranchia & Trochomorphidae & $\begin{array}{l}\text { Geotrochus bicolor } \\
\text { (Martens, 1864) }\end{array}$ & + & \\
\hline 160 & Heterobranchia & Trochomorphidae & $\begin{array}{l}\text { Geotrochus conus } \\
\text { (Pfeiffer, 1841)* }\end{array}$ & + & \\
\hline 161 & Heterobranchia & Trochomorphidae & $\begin{array}{l}\text { Geotrochus multicarinatus } \\
\text { (Böttger, 1890)* }\end{array}$ & + & \\
\hline 162 & Heterobranchia & Trochomorphidae & $\begin{array}{l}\text { Trochomorpha concolor } \\
\text { Böttger, } 1890^{*}\end{array}$ & + & \\
\hline 163 & Heterobranchia & Trochomorphidae & $\begin{array}{l}\text { Trochomorpha froggatti } \\
\text { (Iredale, 1941) }\end{array}$ & + & + \\
\hline 164 & Heterobranchia & Trochomorphidae & $\begin{array}{l}\text { Trochomorpha strubelli } \\
\text { Böttger, } 1890 *\end{array}$ & + & + \\
\hline 165 & Heterobranchia & Chronidae & $\begin{array}{l}\text { Kaliella barrakporensis } \\
\text { (Pfeiffer, 1853) }\end{array}$ & + & \\
\hline 166 & Heterobranchia & Chronidae & $\begin{array}{l}\text { Kaliella dendrophila } \\
\text { (van Benthem Jutting, 1950) }\end{array}$ & + & \\
\hline 167 & Heterobranchia & Chronidae & $\begin{array}{l}\text { Kaliella doliolum } \\
\text { (Pfeiffer, 1846) }\end{array}$ & + & + \\
\hline 168 & Heterobranchia & Chronidae & $\begin{array}{l}\text { Kaliella microconus } \\
\text { (Mousson, 1865) }\end{array}$ & + & + \\
\hline 169 & Heterobranchia & Chronidae & $\begin{array}{l}\text { Kaliella micula } \\
\text { (Mousson, 1857) }\end{array}$ & + & \\
\hline 170 & Heterobranchia & Chronidae & $\begin{array}{l}\text { Kaliella platyconus } \\
\text { Möllendorff, } 1897\end{array}$ & + & + \\
\hline 171 & Heterobranchia & Chronidae & $\begin{array}{l}\text { Kaliella scandens } \\
(\text { Cox, 1872) }\end{array}$ & + & + \\
\hline 172 & Heterobranchia & Chronidae & $\begin{array}{l}\text { Vitrinopsis fruhstorferi } \\
\text { (Möllendorff, 1897) }\end{array}$ & + & \\
\hline 173 & Heterobranchia & Dyakiidae & $\begin{array}{l}\text { Dyakia clypeus } \\
\text { (Mousson, 1857) }\end{array}$ & + & \\
\hline 174 & Heterobranchia & Dyakiidae & $\begin{array}{l}\text { Dyakia rumphii } \\
\text { (Pfeiffer, 1842) }\end{array}$ & + & \\
\hline 175 & Heterobranchia & Dyakiidae & $\begin{array}{l}\text { Elaphroconcha bataviana } \\
\text { (Pfeiffer, 1842) }\end{array}$ & + & + \\
\hline 176 & Heterobranchia & Dyakiidae & $\begin{array}{l}\text { Elaphroconcha javacensis } \\
\text { (Férussac, 1821) }\end{array}$ & + & + \\
\hline 177 & Heterobranchia & Dyakiidae & $\begin{array}{l}\text { Elaphroconcha patens } \\
\text { (Martens, 1898)* }\end{array}$ & + & \\
\hline 178 & Heterobranchia & Dyakiidae & $\begin{array}{l}\text { Inozonites imitator } \\
\text { Möllendorff, } 1897^{*}\end{array}$ & + & \\
\hline 179 & Heterobranchia & Euconulidae & $\begin{array}{l}\text { Coneuplecta macrostoma } \\
\text { (Möllendorff, 1897)* }\end{array}$ & + & \\
\hline 180 & Heterobranchia & Euconulidae & $\begin{array}{l}\text { Coneuplecta olivacea } \\
\text { Vermeulen, } 1996\end{array}$ & + & + \\
\hline 181 & Heterobranchia & Euconulidae & $\begin{array}{l}\text { Coneuplecta sitaliformis } \\
\text { (Möllendorff, 1897) }\end{array}$ & + & + \\
\hline 182 & Heterobranchia & Euconulidae & $\begin{array}{l}\text { Guppya gundlachi } \\
\text { (Pfeiffer, 1840)** }\end{array}$ & + & \\
\hline
\end{tabular}


Table 1. (continued)

\begin{tabular}{|c|c|c|c|c|c|}
\hline \multirow{2}{*}{ No } & \multirow{2}{*}{ Subclass } & \multirow{2}{*}{ Family } & \multirow{2}{*}{ Species } & \multicolumn{2}{|c|}{ Distribution } \\
\hline & & & & Java & $\begin{array}{l}\text { Adjacent } \\
\text { Islands }\end{array}$ \\
\hline 183 & Heterobranchia & Euconulidae & $\begin{array}{l}\text { Liardetia acutiuscula } \\
\text { (Möllendorff, 1897) }\end{array}$ & + & \\
\hline 184 & Heterobranchia & Euconulidae & $\begin{array}{l}\text { Liardetia convexiconica } \\
\text { (Möllendorff, 1897) }\end{array}$ & + & + \\
\hline 185 & Heterobranchia & Euconulidae & $\begin{array}{l}\text { Liardetia densetorta } \\
\text { (Möllendorff, 1897) }\end{array}$ & + & \\
\hline 186 & Heterobranchia & Euconulidae & $\begin{array}{l}\text { Liardetia javana } \\
\text { (Böttger, 1890) }\end{array}$ & + & \\
\hline 187 & Heterobranchia & Euconulidae & $\begin{array}{l}\text { Liardetia pisum } \\
\text { (Möllendorff, 1897)* }\end{array}$ & + & \\
\hline 188 & Heterobranchia & Euconulidae & $\begin{array}{l}\text { Liardetia reticulata } \\
\text { van Benthem Jutting, } 1950\end{array}$ & + & \\
\hline 189 & Heterobranchia & Euconulidae & $\begin{array}{l}\text { Liardetia viridula } \\
\text { (Möllendorff, 1897) }\end{array}$ & + & \\
\hline 190 & Heterobranchia & Euconulidae & $\begin{array}{l}\text { Lamprocystis gedeana } \\
\text { Möllendorff, 1897* }\end{array}$ & + & \\
\hline 191 & Heterobranchia & Helicarionidae & $\begin{array}{l}\text { Durgella pusilla } \\
\text { (Martens, 1867) }\end{array}$ & + & \\
\hline 192 & Heterobranchia & Helicarionidae & $\begin{array}{l}\text { Durgella sundana } \\
\text { Rensch, } 1930\end{array}$ & + & \\
\hline 193 & Heterobranchia & Helicarionidae & $\begin{array}{l}\text { "Helicarion" albellus } \\
\text { Martens, } 1867\end{array}$ & + & + \\
\hline 194 & Heterobranchia & Helicarionidae & $\begin{array}{l}\text { "Helicarion” perfragilis } \\
\text { Möllendorff, } 1897\end{array}$ & + & \\
\hline 195 & Heterobranchia & Helicarionidae & $\begin{array}{l}\text { "Helicarion" radiatulus } \\
\text { (Möllendorff, 1897) }\end{array}$ & + & \\
\hline 196 & Heterobranchia & Ariophantidae & $\begin{array}{l}\text { Hemiplecta humphreysiana } \\
\text { (Lea, 1840) }\end{array}$ & + & + \\
\hline 197 & Heterobranchia & Ariophantidae & $\begin{array}{l}\text { Hemiplecta kangeanensis } \\
\text { Schepman, 1913* }\end{array}$ & & + \\
\hline 198 & Heterobranchia & Ariophantidae & $\begin{array}{l}\text { Macrochlamys amboinensis } \\
\text { (Martens, 1864)** }\end{array}$ & + & \\
\hline 199 & Heterobranchia & Ariophantidae & $\begin{array}{l}\text { Macrochlamys infans } \\
\text { (Reeve, 1854) }\end{array}$ & + & \\
\hline 200 & Heterobranchia & Ariophantidae & $\begin{array}{l}\text { Macrochlamys spiralifer } \\
\text { Vermeulen, } 1996\end{array}$ & + & \\
\hline 201 & Heterobranchia & Ariophantidae & $\begin{array}{l}\text { Microcystina chionodiscus } \\
\text { Vermeulen, } 1996\end{array}$ & + & + \\
\hline 202 & Heterobranchia & Ariophantidae & $\begin{array}{l}\text { Microcystina circumlineata } \\
\text { (Möllendorff, 1897) }\end{array}$ & + & + \\
\hline 203 & Heterobranchia & Ariophantidae & $\begin{array}{l}\text { Microcystina exigua } \\
\text { (Möllendorff, 1897) }\end{array}$ & + & \\
\hline 204 & Heterobranchia & Ariophantidae & $\begin{array}{l}\text { Microcystina fruhstorferi } \\
\text { (Möllendorff, 1897)* }\end{array}$ & + & \\
\hline 205 & Heterobranchia & Ariophantidae & $\begin{array}{l}\text { Microcystina gratilla } \\
\text { van Benthem Jutting, } 1950\end{array}$ & + & + \\
\hline 206 & Heterobranchia & Ariophantidae & $\begin{array}{l}\text { Microcystina muscorum } \\
\text { van Benthem Jutting, } 1959\end{array}$ & + & + \\
\hline
\end{tabular}


Table 1. (continued)

\begin{tabular}{|c|c|c|c|c|c|}
\hline \multirow{2}{*}{ No } & \multirow{2}{*}{ Subclass } & \multirow{2}{*}{ Family } & \multirow{2}{*}{ Species } & \multicolumn{2}{|c|}{ Distribution } \\
\hline & & & & Java & $\begin{array}{l}\text { Adjacent } \\
\text { Islands }\end{array}$ \\
\hline 207 & Heterobranchia & Ariophantidae & $\begin{array}{l}\text { Microcystina nana } \\
\text { (Möllendorff, 1897) }\end{array}$ & + & + \\
\hline 208 & Heterobranchia & Ariophantidae & $\begin{array}{l}\text { Microcystina sinica } \\
\text { Möllendorff, } 1885\end{array}$ & + & + \\
\hline 209 & Heterobranchia & Ariophantidae & $\begin{array}{l}\text { Microcystina subglobosa } \\
\text { (Möllendorff, 1897)* }\end{array}$ & + & + \\
\hline 210 & Heterobranchia & Ariophantidae & $\begin{array}{l}\text { Microcystina vitreiformis } \\
\text { (Möllendorff, 1897) }\end{array}$ & + & + \\
\hline 211 & Heterobranchia & Ariophantidae & $\begin{array}{l}\text { Microparmarion austeni } \\
\text { Simroth, } 1893^{*}\end{array}$ & + & \\
\hline 212 & Heterobranchia & Ariophantidae & $\begin{array}{l}\text { Microparmarion strubelli } \\
\text { Simroth, } 1893\end{array}$ & + & \\
\hline 213 & Heterobranchia & Ariophantidae & $\begin{array}{l}\text { Parmarion martensi } \\
\text { Simroth, } 1893\end{array}$ & + & \\
\hline 214 & Heterobranchia & Ariophantidae & $\begin{array}{l}\text { Parmarion pupillaris } \\
\text { (Humbert, 1829) }\end{array}$ & + & \\
\hline 215 & Heterobranchia & Camaenidae & $\begin{array}{l}\text { Amphidromus alticola } \\
\text { Fulton, 1896* }\end{array}$ & + & \\
\hline 216 & Heterobranchia & Camaenidae & $\begin{array}{l}\text { Amphidromus banksi } \\
\text { Butot, 1954* }\end{array}$ & & + \\
\hline 217 & Heterobranchia & Camaenidae & $\begin{array}{l}\text { Amphidromus filozonatus } \\
\text { (Martens, 1867)* }\end{array}$ & + & + \\
\hline 218 & Heterobranchia & Camaenidae & $\begin{array}{l}\text { Amphidromus furcillatus } \\
\text { (Mousson, 1849) }\end{array}$ & + & + \\
\hline 219 & Heterobranchia & Camaenidae & $\begin{array}{l}\text { Amphidromus heerianus } \\
\text { (Pfeiffer, 1871)* }\end{array}$ & + & + \\
\hline 220 & Heterobranchia & Camaenidae & $\begin{array}{l}\text { Amphidromus javanicus } \\
\text { (Sowerby, 1833)* }\end{array}$ & + & \\
\hline 221 & Heterobranchia & Camaenidae & $\begin{array}{l}\text { Amphidromus jeffabbasorum } \\
\text { Thach, 2016* }\end{array}$ & + & \\
\hline 222 & Heterobranchia & Camaenidae & $\begin{array}{l}\text { Amphidromus palaceus } \\
\text { (Mousson, 1849) }\end{array}$ & + & \\
\hline 223 & Heterobranchia & Camaenidae & $\begin{array}{l}\text { Amphidromus perversus } \\
\text { (Linnaeus, 1758) }\end{array}$ & + & + \\
\hline 224 & Heterobranchia & Camaenidae & $\begin{array}{l}\text { Amphidromus porcellanus } \\
\text { (Mousson, 1849)* }\end{array}$ & + & \\
\hline 225 & Heterobranchia & Camaenidae & $\begin{array}{l}\text { Amphidromus sancangensis } \\
\text { Dharma, 2007* }\end{array}$ & + & \\
\hline 226 & Heterobranchia & Camaenidae & $\begin{array}{l}\text { Amphidromus winteri } \\
\text { (Pfeiffer, 1849)* }\end{array}$ & + & \\
\hline 227 & Heterobranchia & Camaenidae & $\begin{array}{l}\text { Bradybaena similaris } \\
\text { (Férussac, } 1821)^{* *}\end{array}$ & + & + \\
\hline 228 & Heterobranchia & Camaenidae & $\begin{array}{l}\text { Chloritis crassula } \\
\text { (Philippi, 1844)* }^{*}\end{array}$ & + & \\
\hline 229 & Heterobranchia & Camaenidae & $\begin{array}{l}\text { Chloritis fruhstorferi } \\
\text { Möllendorff, } 1897^{*}\end{array}$ & + & + \\
\hline 230 & Heterobranchia & Camaenidae & $\begin{array}{l}\text { Chloritis helicinoides } \\
\text { (Mousson, 1848)* }\end{array}$ & + & + \\
\hline
\end{tabular}


Table 1. (continued)

\begin{tabular}{|c|c|c|c|c|c|}
\hline \multirow{2}{*}{ No } & \multirow{2}{*}{ Subclass } & \multirow{2}{*}{ Family } & \multirow{2}{*}{ Species } & \multicolumn{2}{|c|}{ Distribution } \\
\hline & & & & Java & $\begin{array}{l}\text { Adjacent } \\
\text { Islands }\end{array}$ \\
\hline 231 & Heterobranchia & Camaenidae & $\begin{array}{l}\text { Chloritis transversalis } \\
\text { (Mousson, 1857) }\end{array}$ & + & + \\
\hline 232 & Heterobranchia & Camaenidae & $\begin{array}{l}\text { Ganesella bantamensis } \\
\text { (Smith, 1887)* }\end{array}$ & + & \\
\hline 233 & Heterobranchia & Camaenidae & $\begin{array}{l}\text { Landouria abdidalem } \\
\text { Nurinsiyah, Neiber \& Hausdorf, 2019* }\end{array}$ & + & \\
\hline 234 & Heterobranchia & Camaenidae & $\begin{array}{l}\text { Landouria ciliocincta } \\
\text { (Möllendorff, 1897)* }^{*}\end{array}$ & + & \\
\hline 235 & Heterobranchia & Camaenidae & $\begin{array}{l}\text { Landouria conoidea } \\
\text { (Leschke, 1914) }\end{array}$ & + & \\
\hline 236 & Heterobranchia & Camaenidae & $\begin{array}{l}\text { Landouria davini } \\
\text { Dharma, 2015* }\end{array}$ & + & \\
\hline 237 & Heterobranchia & Camaenidae & $\begin{array}{l}\text { Landouria dharmai } \\
\text { Nurinsiyah, Neiber \& Hausdorf, } 2019\end{array}$ & + & \\
\hline 238 & Heterobranchia & Camaenidae & $\begin{array}{l}\text { Landouria epiplatia } \\
\text { (Möllendorff, 1897)* }\end{array}$ & + & \\
\hline 239 & Heterobranchia & Camaenidae & $\begin{array}{l}\text { Landouria intumescens } \\
\text { (Martens, 1867)* }\end{array}$ & + & \\
\hline 240 & Heterobranchia & Camaenidae & $\begin{array}{l}\text { Landouria kangeanensis } \\
\text { (Schepman, 1913)* }\end{array}$ & & + \\
\hline 241 & Heterobranchia & Camaenidae & $\begin{array}{l}\text { Landouria leucochila } \\
\text { (Gude, 1905)* }\end{array}$ & + & \\
\hline 242 & Heterobranchia & Camaenidae & $\begin{array}{l}\text { Landouria madurensis } \\
\text { Nurinsiyah, Neiber \& Hausdorf, 2019* }\end{array}$ & & + \\
\hline 243 & Heterobranchia & Camaenidae & $\begin{array}{l}\text { Landouria menorehensis } \\
\text { Nurinsiyah, Neiber \& Hausdorf, 2019* }\end{array}$ & + & \\
\hline 244 & Heterobranchia & Camaenidae & $\begin{array}{l}\text { Landouria monticola } \\
\text { van Benthem Jutting, 1950* }\end{array}$ & + & \\
\hline 245 & Heterobranchia & Camaenidae & $\begin{array}{l}\text { Landouria moussoniana } \\
\text { (Martens, 1867)* }\end{array}$ & + & \\
\hline 246 & Heterobranchia & Camaenidae & $\begin{array}{l}\text { Landouria naggsi } \\
\text { Nurinsiyah, Neiber \& Hausdorf, 2019* }\end{array}$ & + & \\
\hline 247 & Heterobranchia & Camaenidae & $\begin{array}{l}\text { Landouria nodifera } \\
\text { Nurinsiyah, Neiber \& Hausdorf, 2019* }\end{array}$ & + & \\
\hline 248 & Heterobranchia & Camaenidae & $\begin{array}{l}\text { Landouria nusakambangensis } \\
\text { Nurinsiyah, Neiber \& Hausdorf, 2019* }\end{array}$ & & + \\
\hline 249 & Heterobranchia & Camaenidae & $\begin{array}{l}\text { Landouria pacitanensis } \\
\text { Nurinsiyah, Neiber \& Hausdorf, 2019* }\end{array}$ & + & \\
\hline 250 & Heterobranchia & Camaenidae & $\begin{array}{l}\text { Landouria pakidulan } \\
\text { Nurinsiyah, Neiber \& Hausdorf, 2019* }\end{array}$ & + & + \\
\hline 251 & Heterobranchia & Camaenidae & $\begin{array}{l}\text { Landouria parahyangensis } \\
\text { Nurinsiyah, Neiber \& Hausdorf, 2019* }\end{array}$ & + & \\
\hline 252 & Heterobranchia & Camaenidae & $\begin{array}{l}\text { Landouria petrukensis } \\
\text { Nurinsiyah, Neiber \& Hausdorf, 2019* }\end{array}$ & + & \\
\hline 253 & Heterobranchia & Camaenidae & $\begin{array}{l}\text { Landouria rotatoria } \\
\text { (Pfeiffer, 1842)* }\end{array}$ & + & \\
\hline 254 & Heterobranchia & Camaenidae & $\begin{array}{l}\text { Landouria schepmani } \\
\text { (Möllendorff, 1897)* }\end{array}$ & + & \\
\hline
\end{tabular}


Table 1. (continued)

\begin{tabular}{|c|c|c|c|c|c|}
\hline \multirow{2}{*}{ No } & \multirow{2}{*}{ Subclass } & \multirow{2}{*}{ Family } & \multirow{2}{*}{ Species } & \multicolumn{2}{|c|}{ Distribution } \\
\hline & & & & Java & $\begin{array}{c}\text { Adjacent } \\
\text { Islands }\end{array}$ \\
\hline 255 & Heterobranchia & Camaenidae & $\begin{array}{l}\text { Landouria sewuensis } \\
\text { Nurinsiyah, Neiber \& Hausdorf, 2019* }\end{array}$ & + & \\
\hline 256 & Heterobranchia & Camaenidae & $\begin{array}{l}\text { Landouria smimensis } \\
\text { (Mousson, 1848)* }\end{array}$ & + & \\
\hline 257 & Heterobranchia & Camaenidae & $\begin{array}{l}\text { Landouria sukoliloensis } \\
\text { Nurinsiyah, Neiber \& Hausdorf, 2019* }\end{array}$ & + & \\
\hline 258 & Heterobranchia & Camaenidae & $\begin{array}{l}\text { Landouria tholiformis } \\
\text { Nurinsiyah, Neiber \& Hausdorf, 2019* }\end{array}$ & + & \\
\hline 259 & Heterobranchia & Camaenidae & $\begin{array}{l}\text { Landouria tonywhitteni } \\
\text { Nurinsiyah, Neiber \& Hausdorf, 2019* }\end{array}$ & + & \\
\hline 260 & Heterobranchia & Camaenidae & $\begin{array}{l}\text { Landouria winteriana } \\
\text { (Pfeiffer, 1842) }\end{array}$ & + & \\
\hline 261 & Heterobranchia & Camaenidae & $\begin{array}{l}\text { Landouria zonifera } \\
\text { Nurinsiyah, Neiber \& Hausdorf, 2019* }\end{array}$ & + & \\
\hline 262 & Heterobranchia & Camaenidae & $\begin{array}{l}\text { Pseudopartula arborascens } \\
\text { Butot, } 1955^{*}\end{array}$ & + & + \\
\hline 263 & Heterobranchia & Camaenidae & $\begin{array}{l}\text { Pseudopartula galericulum } \\
\text { (Mousson, 1849) }\end{array}$ & + & \\
\hline
\end{tabular}

Many of the endemic species in Java are restricted to a small region. Among the total number of species that occur in Java, 53\% (139 species) were recorded in karst areas. Twenty-five species occur exclusively in karst areas. Each of the fourteen of these karstexclusive species were each found only in a single karst area. For instance, among 27 Landouria species in Java, 21 species have restricted distributions and are only recorded in small range areas in Java (Nurinsiyah et al., 2019). From 23 diplommatinids, 12 species were recorded only in non-karst (volcanic/mountain) areas. The records of these small range endemic forest species might indicate that small scale forest refugia probably existed during the glacial period.

\section{DISCUSSION}

Based on the extremely high species richness of plants and vertebrates, Indonesia is listed as one of the mega diversity countries (Reid, 1998). Furthermore, the country is also included among the 25 biodiversity hotspots (Sundaland) based on the number of endemic species and the degree of threat (Myers et al., 2000). Fourteen percent of the land snail species known in Indonesia occur in Java (Hausdorf, 2019). The number of land snail species in Java is slightly differ from Sumatra and Sulawesi, which have larger areas (Table 2). The land snail species diversity in the two latter islands might be underestimated due to 
the lack of malacological study in these areas. This is the case not only for snails, but also for other invertebrate taxa that are often neglected.

Table 2. Land snail species diversity in the archipelago (source: adapted from Hausdorf, 2019)

\begin{tabular}{lcc}
\hline \multicolumn{1}{c}{ Island } & Area $\left(\mathbf{k m}^{\mathbf{2}}\right)$ & Number of Species \\
\hline Sumatra & 473,481 & 276 \\
Java \& adjacent islands & 129,438 & 263 \\
Bali \& Nusa Penida & 5,780 & 126 \\
Borneo & 743,330 & 558 \\
Sulawesi & 180,680 & 253 \\
\hline
\end{tabular}

The 263 land species in Java and adjacent islands is a great increment from the previous comprehensive list which was 171 land snail species (van Benthem Jutting, 1941, 1948, 1950, 1952). The additional species came from new record of native species, new record of introduced species, and newly described species. In the past two decades there were 26 new species of Gastropods have been described from Java. There were also fossil species of Gastropods described (Dharma, 2007, 2021) but they are not included in the list. Whitten et al. (1997) listed seven introduced species from Java i.e. Allopeas gracile, Lissachatina fulica, Subulina octona, Gulella bicolor, Rachis zonulata, Gastrocopta servilis, and Bradybaena similaris. The number was added to 13 introduced species (Nurinsiyah \& Hausdorf, 2019). The widely distributed introduced species occur mainly in the disturbed areas and modified habitats. The proportion of introduced species abundance in an area can be use as indicator for habitat disturbance (Nurinsiyah et al., 2016).

Among the 17 land snail species found on the adjacent islands (not in mainland), nine species are endemic to a particular island. The species of Geophorus rollei, Cyclotus kangeanus, Hemiplecta kangeanensis, and Landouria kangeanensis were found only in Kangean island (van Benthem Jutting, 1941). The island is located more than $120 \mathrm{~km}$ from the mainland Java even though administratively belongs to the Province of East Java. There are also species endemic to adjacent island located less than $1 \mathrm{~km}$ away from the mainland Java. Dicharax candrakirana is endemic to Sempu Is. and Landouria nusakambangensis is endemic to Nusa Kambangan Is. (Nurinsiyah \& Hausdorf, 2017b; Nurinsiyah et al., 2019). The former island is a nature reserve, whereas the latter is a prison island which public have limited access to enter the area. However, there is a concession area for limestone quarry in the northern part of the Nusa Kambangan Is. Although more than $50 \%$ of the concession area is designated for conservation forest, land coverage and habitat sustainability for the forest inhabitants needs to be ensured. 
Terrestrial mollusk is one of the fauna groups that is often overlooked and abandoned from the conservation spotlight. It was estimated that this group may have lost $7 \%$ of the total described species on Earth (Régnier et al., 2015). Although the work of land snails in Java is the most complete compared to other islands in the Indonesian archipelago, monitoring the sustain population in Java is crucial. Furthermore, there will always be a yet discovered taxa or systematically problematic taxa in this well-known island awaiting scientific discovery.

\section{ACKNOWLEDGEMENTS}

I am grateful to Prof. Bernhard Hausdorf (Centrum für Naturkunde, Universität Hamburg) for supervising the research; Tedi Setiadi for the help in this study; Alfiah, Heryanto, N. Isnaningsih, R. Marwoto, N. Mujiono, and R. Prihandini from the Museum Zoologicum Bogoriense Indonesia for providing resources and help in laboratory work; A.F. Bakti (Bandung), H. Cahyono (Malang), I. Faizah (Lebak), H. Fauzia (Malang), F. Irsyad (Bandung), Kaspo (Yogyakarta), H. Mada (Bandung), R. Nasrullah (Tasikmalaya), E. Nurlela (Bandung), Y. Prasetio (Kabandungan), M. Rizky (Tasikmalaya), and F. Sari (Bandung) for assistance in field and laboratory work. I also thank Barna Páll-Gergely, Thor Seng Liew, and Gizelle Batomalaque for the comments and suggestions for the improvement of this manuscript. The study was supported by the Indonesian-German Scholarship Program (KEMENDIKTIRISTEK-RI and DAAD), the University of Hamburg, the Conchologist of America, and BMBF (INDOBIOSYS MfN Berlin, 16GW0111K).

\section{REFERENCES}

Badan Pusat Statistik (BPS). 2017. https://www.bps.go.id/statictable/2014/09/05/1366/luas-daerahdan-jumlah-pulau-menurut-provinsi-2002-2016.html

Bouchet, P., Rocroi, J.P., Hausdorf, B., Kaim, A., Kano, Y., Nützel, A., Parkhaev, P., Schrödl, M. \& Strong, E.E. 2017. Revised classification, nomenclator and typification of gastropod and monoplacophoran families. Malacologia, 61(1-2): 1-526.

Butot, L. 1955. The molluscs fauna of Pulau Panaitan (Prinseneiland). Land and freshwater molluscs. Treubia, 23: 70-135.

Cannon, C.H., Morley, R.J. \& Bush, A.B.G. 2009. The current refugial rainforests of Sundaland are unrepresentative of their biogeographic past and highly vulnerable to disturbance. PNAS, 106 (27): 11188-11193.

Dharma, B. 1992. Siput dan Kerang Indonesia II (Indonesian Shells II). Wiesbaden: Verlag Christa Hemmen [Indonesian].

Dharma, B. 2005. Recent \& Fossil Indonesian Shells. Hackenheim: ConchBooks.

Dharma, B. 2007. Report on fossil Amphidromus and description of new species and new subspecies of recent and fossil Amphidromus from Indonesia (Gastropoda, Pulmonata: Camaenidae). Schriften zur Malakozoologie, 23: 45-78.

Dharma, B. 2014. A new species of Landouria Godwin-Austen 1918 from Jawa, Indonesia (Gastropoda, Bradybaenidae). Schriften zur Malakozoologie, 28: 7-12. 
Dharma, B. 2021. New species and subspecies of recent and fossil Amphidromus Albers, 1850 from Java and Sumatra (Gastropoda: Camaenidae). The Festivus, 53(4): 298-306

Douglas, D.D., Brown, D.R. \& Pederson, N. 2013. Land snail diversity can reflect degrees of anthropogenic disturbance. Ecosphere, 4 (2): 1-14.

Gomes, S.R. \& Thomé, J.W. 2004. Diversity and distribution of the Veronicellidae (Gastropoda: Soleolifera) in the Oriental and Australian biogeographical regions. Memoirs of the Queensland Museum, 49: 589-601.

Greke, K. 2019. New species and record of Diplommatina Benson, 1849 (Gastropoda: Diplommatinidae) from Java, Indonesia. Nautilus, 133: 14-21.

Hausdorf, B. 2019. Beyond Wallace's line - dispersal of Oriental and Australo-Papuan Land-Snails across the Indo-Australian Archipelago. Zoological Journal of the Linnean Society, 185: 66-76.

Heryanto. 2008. Ekologi keong darat di Taman Nasional Gunung Ciremai. Jurnal Biologi Indonesia, 4: 359-370.

Heryanto. 2011. Land Snails of Java. Bogor: LIPI Press [Indonesian].

Heryanto, 2012. Keanekaragaman keong darat di dua macam habitat makro di Gunung Slamet Jawa Tengah. In: Maryanto, I., Noerdjito, M. \& Partomihardjo, T., eds. Ekologi Gunung Slamet: Geologi, Klimatologi, Biodiversitas dan Dinamika Sosial. Jakarta: LIPI Press [Indonesian].

Heryanto. 2017. The Land snail diversity in succession forest of Mount Galunggung and stable forest of Mount Sawal, West Java. Zoo Indonesia, 26(2): 59-69.

Heryanto, Ristiyanti, M.M., Munandar, A. \& Susilowati, P. 2003. Keong dari Taman Nasional Gunung Halimun, Sebuah Buku Panduan Lapangan. Cibinong: Biodiversity Conservation Project -LIPI-JICA-PHKA [Indonesian].

Hughes, A. 2017. Understanding the drivers of Southeast Asian biodiversity loss. Ecosphere, 8(1): 1 -33 .

Loosjes, F.E., 1953. Monograph of the Indo-Australian Clausiliidae (Gastropoda, Pulmonata, Clausiliidae, Phaedusinae). Beaufortia, 31: 1-226.

Martens, E. von, 1867. Die landschnecken. In: Die Preussische expedition nach Ost-Asien. Nach amtlichen Quellen. Zoologischer Theil. Vol. 2. Berlin: Decker [German].

Marwoto, R.M. 2011. Keong darat dan air tawar dari pulau Nusa Kambangan (Moluska, Gastropoda) (The terrestrial and freshwater snails from Nusa Kambangan Island (Mollusk, Gastropod)). Jurnal Moluska Indonesia, 2(1): 39-47.

Ministry of Environment and Forestry (KHLK). 2016. Statistik Kementrian Lingkungan Hidup dan Kehutanan Tahun. 2015. Jakarta: Pusat Data dan Informasi Kementrian Lingkungan Hidup dan Kehutanan Republik Indonesia [Indonesian].

Möllendorff, O. F. von. 1897. Neue Landschnecken von Java. Nachrichtsblatt der Deutschen Malakozoologischen Gesellschaft, 29 (5/6):57-72 \& (7/8): 89-97.

Mujiono, N. \& Priawandiputra, W. 2020. Land snail from limestone karst in Java: an update with motes on their distribution. IOP Conf. Series: Earth and Environmental Science, 457(012002): 19.

Nurhayati, P.A., Affandi, M. \& Nurinsiyah, A.S. 2021. Diversity and abundance of terrestrial gastropods on the slopes of Mount Arjuna-Welirang, East Java, Indonesia. Biodiversitas, 22(10): 4193-4202.

Nurinsiyah, A.S. 2015. Land snail fauna of the Sukolilo karst in Java (Indonesia). American Conchologist, 43: 30-32.

Nurinsiyah, A.S., Fauzia, H., Hennig, C. \& Hausdorf, B. 2016. Native and introduced land snail species as ecological indicators in different land use types in Java. Ecological Indicators, 70: 557 -565 .

Nurinsiyah, A.S. \& Hausdorf, B. 2017a. Revision of the Diplommatinidae (Gastropoda: Cyclophoroidea) from Java. Zootaxa, 4312: 201-245. 
Nurinsiyah, A.S. \& Hausdorf, B. 2017b. Dicharax (?) candrakirana n. sp. (Gastropoda: Cyclophoridae) from Sempu Island, Indonesia. Zootaxa, 4363(4): 589-591.

Nurinsiyah, A.S. \& Hausdorf, B. 2019. Listing, impact assessment and prioritization of introduced land snail and slug species in Indonesia. Journal of Molluscan Studies, 85: 92-102.

Nurinsiyah, A.S., Neiber, M.T. \& Hausdorf, B. 2019. Revision of the land snail genus Landouria Godwin-Austen, 1918 (Gastropoda, Camaenidae) from Java. European Journal of Taxonomy, 526: $1-73$.

Nurinsiyah, A.S. \& Hausdorf, B. 2020. Changes in the composition of the land snail fauna of Mt. Ciampea, West Java, Indonesia. BIO Web of Conferences, 19: 1-5.

Páll-Gergely, B., Sajan, S., Tripathy, B., Meng, K., Asami, T. \& Ablett, J.D. 2020. Genus-level revision of the Alycaeidae (Gastropoda, Cyclophoroidea), with an annotated species catalogue. Zookeys, 981: 1-220.

Purba, C.P.P., Nanggara, S.G., Ratriyono, M., Apriani, I., Rosalina, L., Sari, N.A. \& Meridian, A.H. 2014. Potret Keadaan Hutan Indonesia Periode 2009-2013. Bogor: Forest Watch Indonesia [Indonesian].

Régnier, C., Achaz, G., Lambert, A., Cowie, R.H., Bouchet, P. \& Fontaine, B. 2015. Mass extinction in poorly known taxa. PNAS, 112(25): 7761-7766.

Reid, W.V. 1998. Biodiversity hotspots. Tree, 13(7): 275-280.

Schilthuizen, M., Liew, T.S., Elahan, B. \& Lackman-Ancrenaz, I. 2005. Effect of karst forest degradation on pulmonated and prosobranch land snail communities in Sabah, Malaysian Borneo. Conservation Biology, 19: 949-954.

van Benthem Jutting, W.S.S. 1941. Non-marine mollusca from the satellite islands surrounding Java. Archives Neérlandaises de Zoologie, 5: 251-348.

van Benthem Jutting, W.S.S. 1948. Systematic studies on the non-marine Mollusca of the IndoAustralian archipelago. I. Critical revision of the Javanese pulmonate land-shells of the families Hydrocenidae, Helicinidae, Cyclophoridae, Pupinidae and Cochlostomatidae. Treubia, 19: 539604.

van Benthem Jutting, W.S.S. 1950. Systematic studies on the non-marine Mollusca of the IndoAustralian archipelago. II. Critical revision of the Javanese pulmonate land-shells of the families Helicarionidae, Pleurodontidae, Fruticicolidae and Streptaxidae. Treubia, 20: 381-505.

van Benthem Jutting, W.S.S. 1952. Systematic studies on the non-marine Mollusca of the IndoAustralian archipelago. III. Critical revision of the Javanese pulmonate land-shells of the families Ellobiidae to Limacidae, with an Appendix on Helicarionidae. Treubia, 21: 291-435.

Vermeulen, J.J. 1996. Notes on terrestrial molluscs of Java, Bali and Nusa Penida. Basteria, 59: 149162.

Vermeulen, J.J. \& Whitten A.J. 1998. Fauna Malesiana. Guide to the Land Snails of Bali. Leiden: Backhuys Publishers.

Whitten, T., Soeriaatmadja, R.E. \& Afiff, S.A. 1997. The Ecology of Java and Bali. Hong Kong: Periplus Editions.

Winter, A.J. de. 1983. Euphaedusa cumingiana (L. Pfeiffer) (Pulmonata, Clausiliidae) new to the fauna of Java (Indonesia). Basteria, 47: 59-60.

Winter, A.J. de. \& Vermeulen, J.J. 1998. A first record of Truncatellina (Pulmonata: Vertiginidae) from SE. Asia: Truncatellina cf. insulivaga (Pilsbry \& Hirase) on Java, Indonesia. Basteria, 62: $269-271$. 